
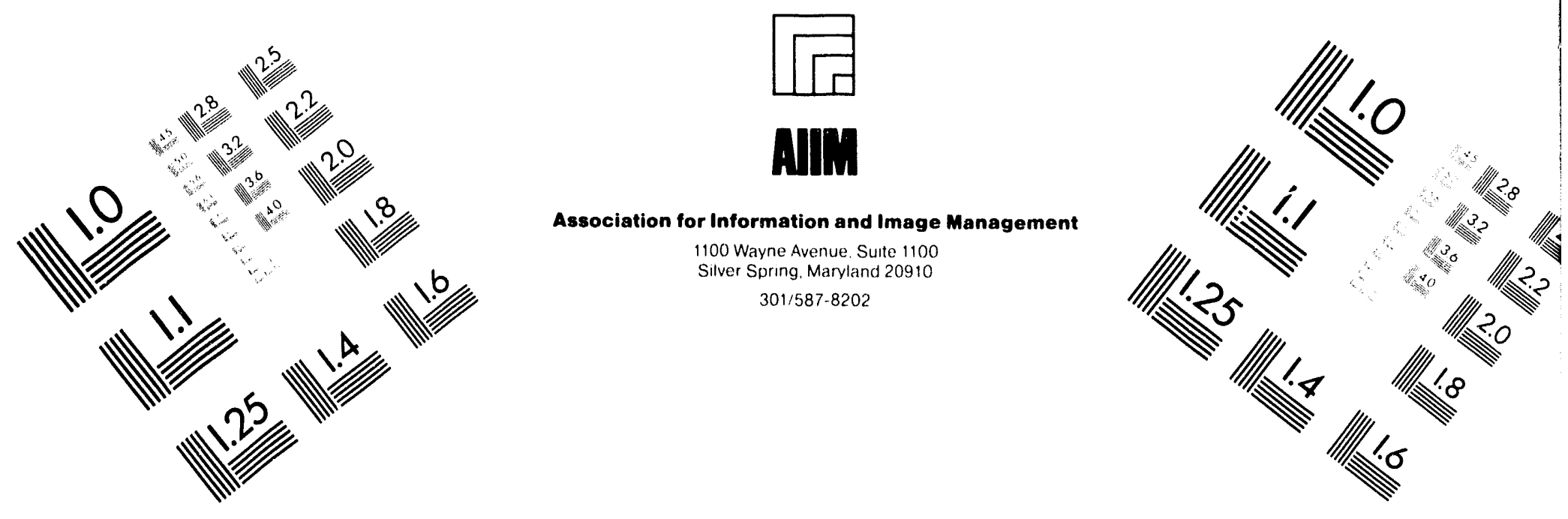

Centimeter

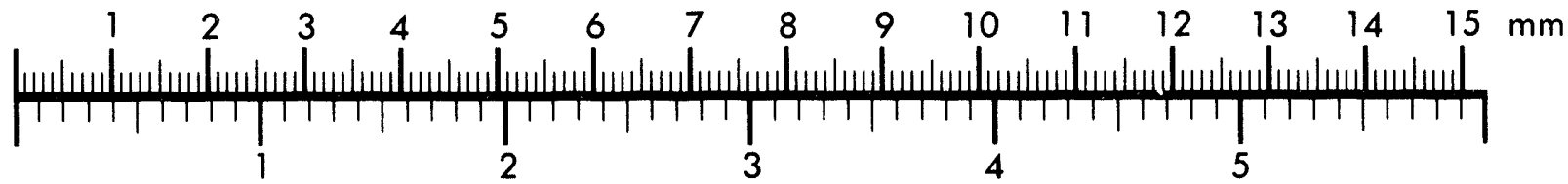
Inches
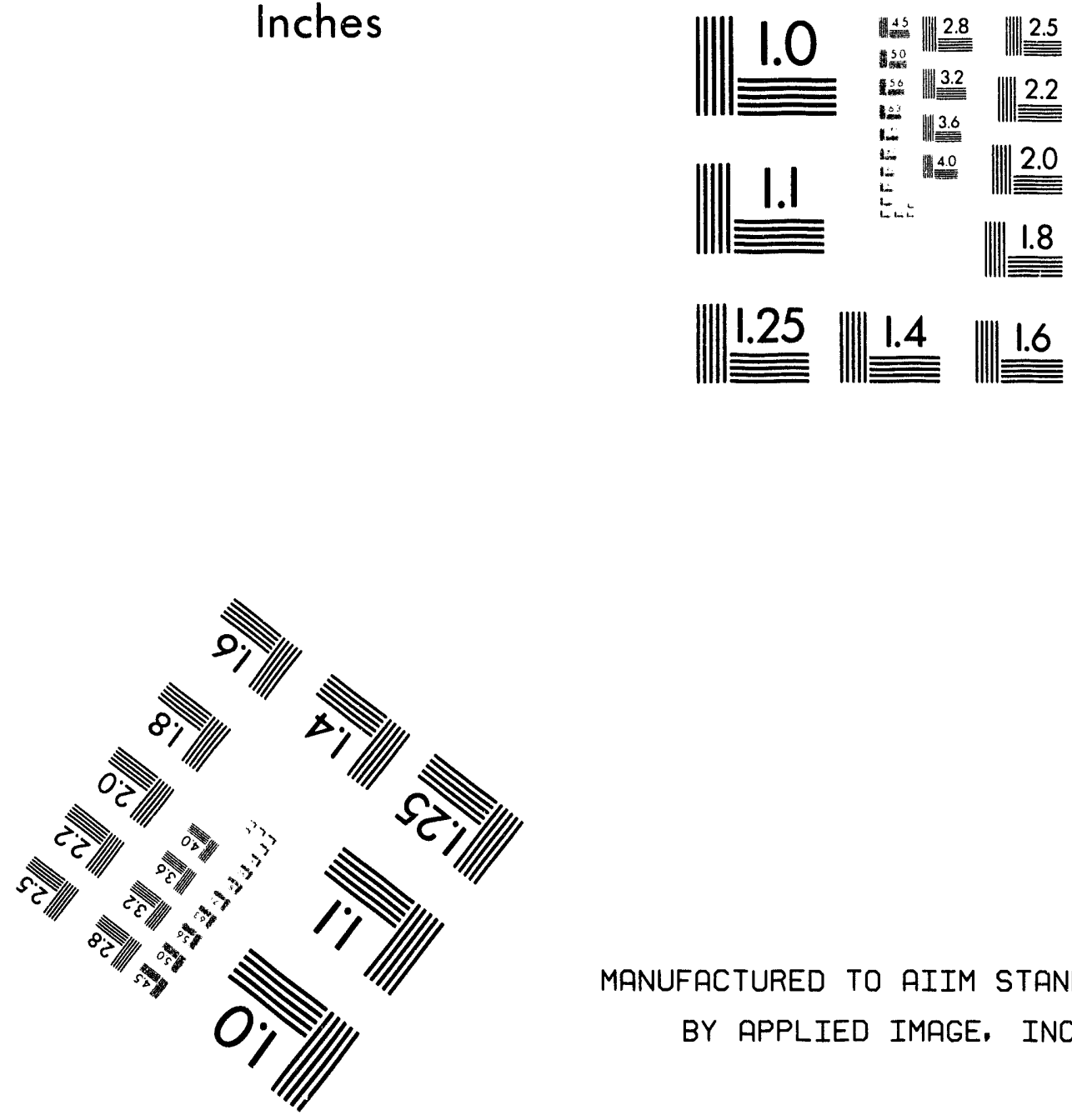

MANUFACTURED TO AIIM STANDARDS

BY APPLIED IMAGE, INC.

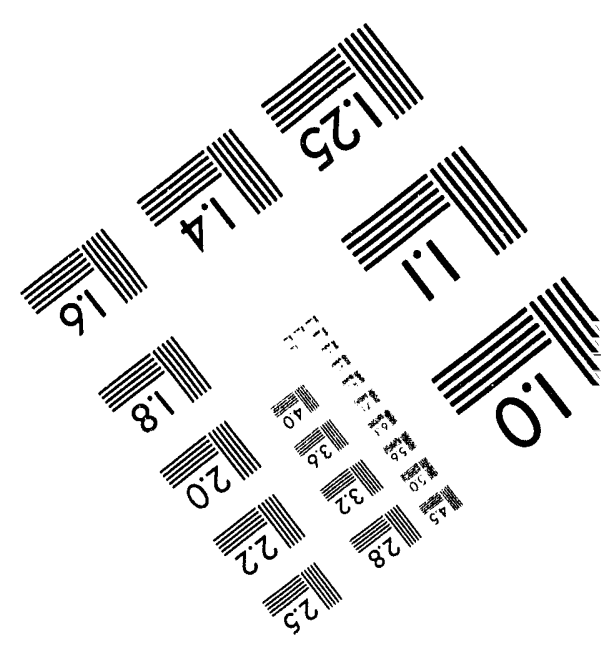



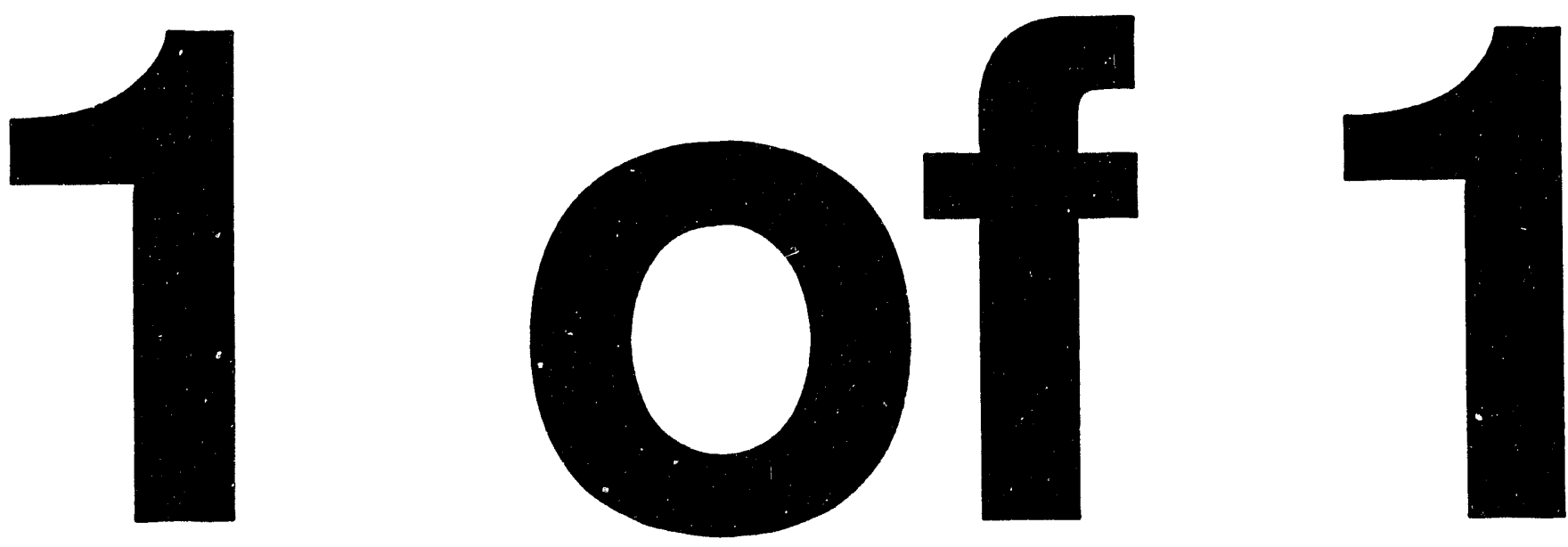
THE LINEARITY PERFORMANCE OF A TWO-

DIMENSIONAL, X-RAY PROPORTIONAL CHAMBER

WITH $0.5 \mathrm{~mm}$ ANODE WIRE SPACING

G.C. Smith and B. Yu

Brookhaven National Laboratory

Upton, NY 11973

\begin{abstract}
A method of construction of multiwire chambers has been developed which yields $X$-ray images with very small non-linearity due to anode wire modulation. The technique requires an absorption region in the detector, with the anode and upper cathode wires, which are parallel to one another, in registration with each other. Studies show that the strength of the electric field in the drift region determines the degree of anode wire modulation and, under specific conditions, modulation is halved in periodicity and significantly reduced in amplitude. New interpolating cathodes have been developed to readout position in the other axis, along the anode wire direction. Position resolution in both axes between 100-150 micron FWHM has been achieved. Twodimensional images of small objects have been taken which show unprecedented linearity.
\end{abstract}

Most suitable topic:

Radiation Detector Systems and Applications

Corresponding Author:

Bo Yu

Building 535B

Brookhaven National Laboratory

Upton, NY 11973-5000

Tel: (516) 282-5184

Fax: (516) 282-5773

E-mail: yubo@bnlux1.bnl.gov

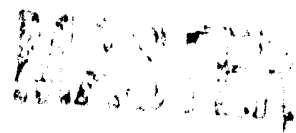




\title{
The Linearity Performance Of A Two-Dimensional, X-ray Proportional Chamber With 0.5mm Anode Wire Spacing
}

\author{
Summary
}

Over the past several years, we have developed 2-D x-ray detectors with delayline readouts for use in small angle scattering experiments [1]. The detectors demonstrate excellent position linearity along the anode wire direction due to optimized designs in several types of interpolating cathodes. However, the position resolution across the anode wires is limited basically to the anode wire pitch ( $1.1 \mathrm{~mm}$ in this case) due to the discrete nature of the anode wire avalanches. Naturally, one can improve the position resolution by decreasing the anode wire spacing. MWPCs with anode wire spacing as small as $0.4 \mathrm{~mm}$ and $0.2 \mathrm{~mm}$ have been built and repnrted [2]. The major disadvantages in this approach are the increase of the detector's high voltage instabilities and the difficulty of maintain a uniform wire spacing.

Recent studies[3] have indicated that in a MWPC with a drift/absorption region, placing the cathode wires in registration with the anode wires and optimizing the electric field strength in the drift region can reduce the amount of anode wire modulation. Based on our previous experience in building two-dimensional $x$-ray MWPCs, we have constructed a new detector with significant reduction in its differential non-linearity due to the anode wire modulation and, therefore, improved position resolution in the direction perpendicular to the anode wires.

The detector has a maximum active area of $2 \mathrm{~cm} \times 10 \mathrm{~cm}$, a $5 \mathrm{~mm}$ deep drift/absorption region. The anode wires are $12 \mu \mathrm{m}$ in diameter, with $0.58 \mathrm{~mm}$ spacing, and located midway between the cathode wire plane and the printed circuit cathode which are separated by $-1.2 \mathrm{~mm}$. The anode and cathode wires are parallel and in registration with each other. Each of the two cathodes is connected to a delayline with $1 \mu$ s delay time. The readout electronics are identical to that described in ref. 1 .

To study the effect of the electric field in the drift region on the anode wire modulation, uniform irradiation responses were recorded across the anode wire direction with different bias voltages on the detector window (Figure 1). Plots of the electron drift lines from the drift region to the anode wires are shown in Figure 2 for the corresponding window voltages. In the case of very weak drift field $\left(\mathrm{V}_{\mathrm{w}}=0\right)$, the drift lines are so heavily pinched in the amplification region that diffusion in the electron cloud results in nearly equal charge sharing between the two adjacent anode wires, irrespective of the $x$-ray absorption position. Recorded $x$-ray positions are then biased to the midway between anode wires. On the other hand, a strong drift field prevents the drift lines from forming a very narrow channel, reducing the amount of charge sharing, resulting in a position bias towards anode wires. When the drift field is optimized, the anode wire modulation has a period half of the anode wire spacing while the amplitude is greatly reduced..

A variety of interpolating cathode patterns have been tested on the printed circuit cathode. Many of them give position resolution better than $120 \mu \mathrm{m}(\mathrm{FWHM})$ with good linearity. On the $10 \mathrm{~cm}$ long cathode, because the ratio between the readout spacing and the anode-cathode spacing is too large $(\sim 4.3)$ for our conventional two intermediate strip (TIS) readout to give a linear response, a new single intermediate zigzag pattern has been developed to provide good linearity and reduced inter-node capacitance. Effects of the angular localization of avalanche charges on the zigzag cathode will be discussed.

Images of small objects taken with this detector have shown unprecedented linearity using $5.4 \mathrm{keV} \mathrm{x}$ rays. Figure 3 shows a comparison between the $x$-ray image of a small gear wheel taken with this detector and that taken with the detector from Ref.1.

References:

1. G.C. Smith et al, Nucl. Instrum. and Method A323 (1992) 78-85.

2. William Frieze et al., Nucl. Instrum. and Method 136 (1976) 93-97.

3. E. Mathieson and G.C. Smith, IEEE Trans. Nucl. Sci. NS-37 (1990) 187-191. 

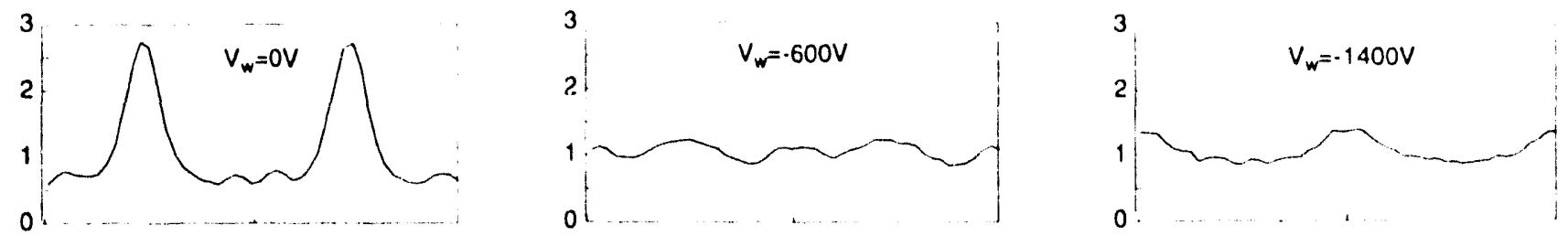

Figure 1. Uniform irradiation responses of the detector across the anode wire direction showing dramatic changes in the anode wire modulation under three different window bias voltages $\left(\mathrm{V}_{\mathrm{w}}\right)$ with a gas mixture of Argon $/ 20 \% \mathrm{CO}_{2}$.
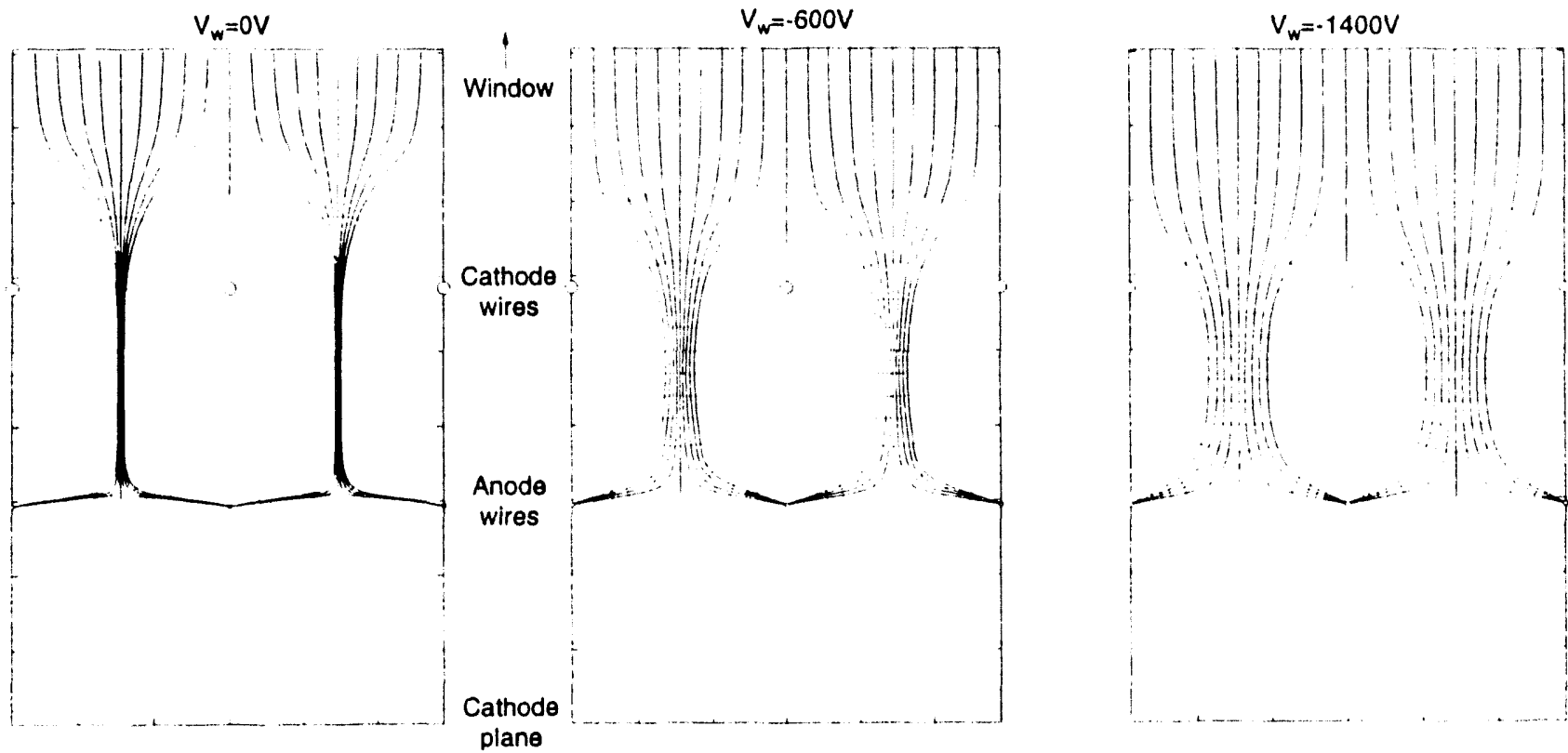

Figure 2. Plots of electron drift lines from the drift region to the anode wires for three different window bias voltages $\left(\mathrm{V}_{\mathrm{w}}\right)$. Both cathode planes are at ground potential, while the anodes are at about $1200 \mathrm{~V}$.
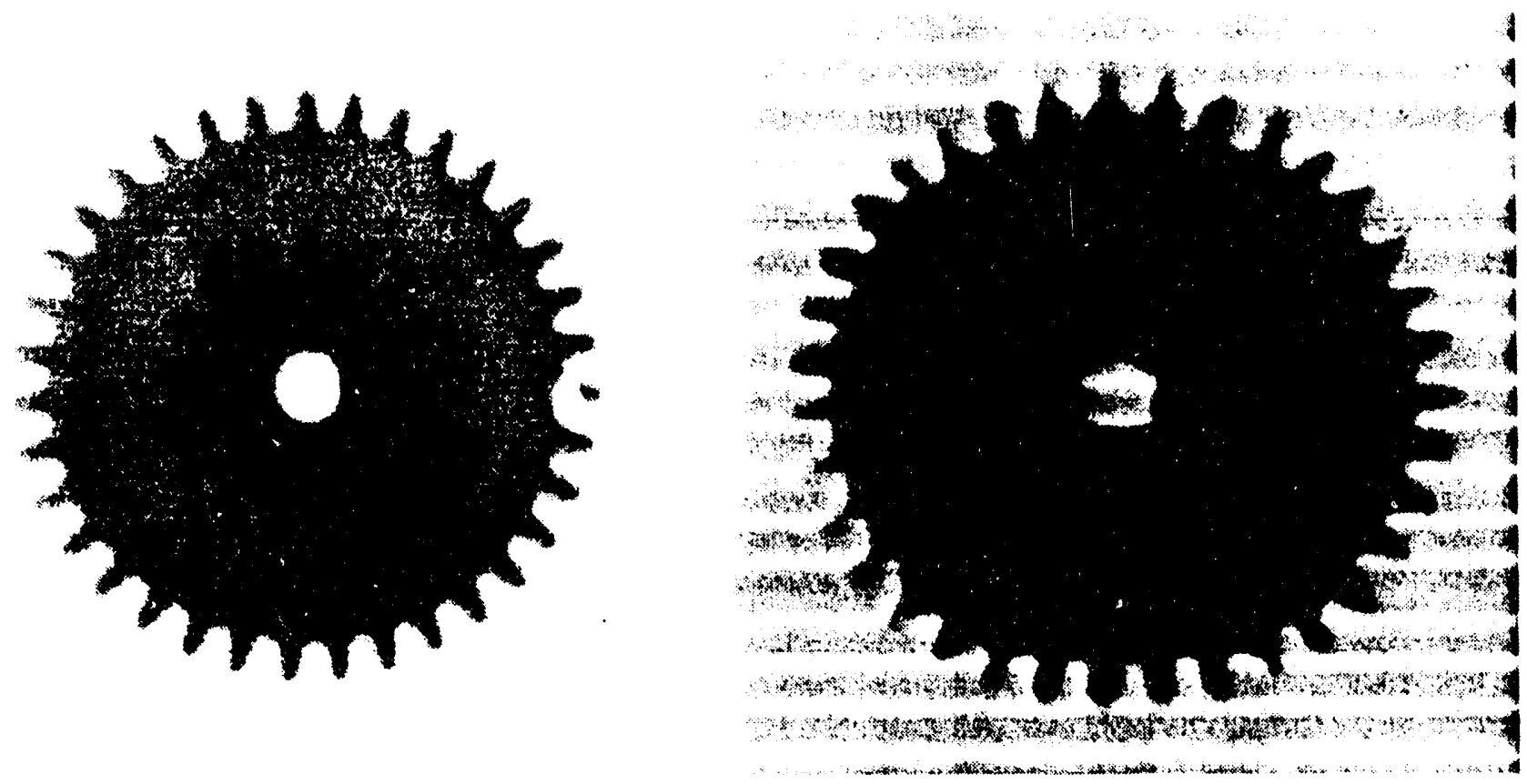

Figure 3. $5.4 \mathrm{keV} x$-ray images of a $15 \mathrm{~mm}$ diameter plastic gear wheel from this detector (left) and another detector with $1.1 \mathrm{~mm}$ anode wire spacing (right). The distance between gear teeth is about $1 \mathrm{~mm}$. 

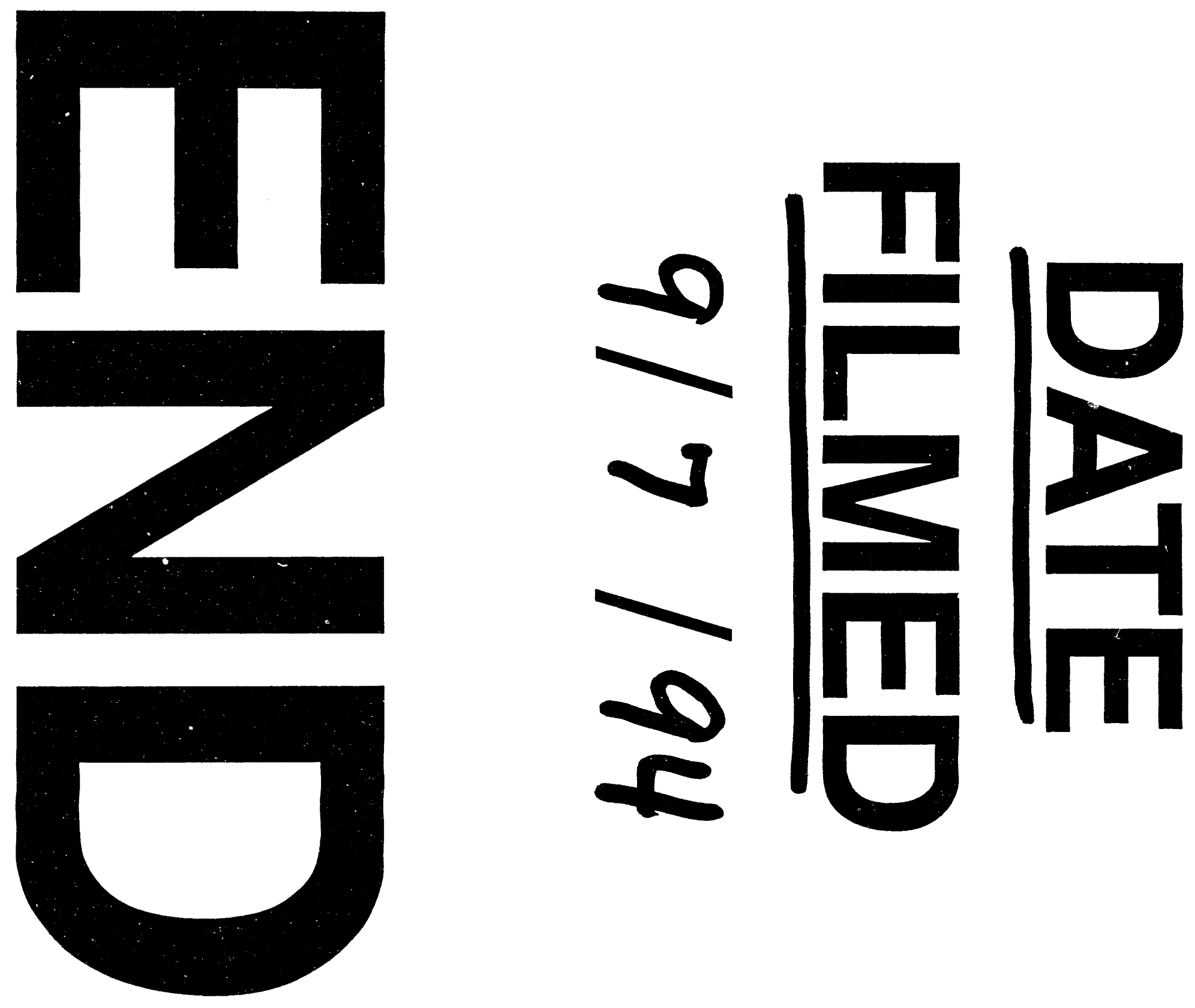


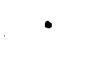

